\title{
Chebyshev pseudospectral collocation method for magneto-nanofluid flow through a porous channel with suction/injection at slowly expanding/contracting walls
}

\author{
Gbeminiyi Sobamowo*, Ahmed Yinusa, Akinbowale Akinshilo \\ University of Lagos, Akoka, Lagos, Nigeria
}

(Received: 2 June 2020. Received in revised form: 21 July 2020. Accepted: 14 August 2020. Published online: 31 August 2020.)

(c) 2020 the authors. This is an open access article under the CC BY (International 4.0) license (www.creativecommons.org/licenses/by/4.0/).

\begin{abstract}
This study analyzes two-dimensional magnetohydrodynamic nanofluid flow through walls with a porous channel of admittance using pseudo-spectral Chebyshev collocation scheme. The magnetohydrodynamic model is developed by incorporating suction/injection in the slowly contracting/expanding walls of the channel. The obtained results are verified with RungeKutta method and some excellent agreements are established. The simulated results through the scheme employed in the present study revealed that the channel lateral velocity decreases with an increase in the flow Reynolds number during expansion. This velocity is also observed to be proportional to the wall expansion ratio with a decreased value towards the porous channel center. As a result of the tremendous accuracy demonstrated by the collocation method, the obtained solutions may serve as a benchmark for the subsequent investigation and studies in this research area.
\end{abstract}

Keywords: magnetohydrodynamics; porous channel; nanofluid; expanding/contracting walls; pseudo-spectral Chebyshev collocation scheme.

2020 Mathematics Subject Classification: 65L10.

\section{Introduction}

Laminar fluid flow through permeable walls with a porous channel of admittance can be seen in various applications of transport and biological fluid flow phenomena such as in the kidneys and lungs internal filtration, porous diaphragms synchronous pulsation, lymphatics internal flow and respiratory system. Various studies have been presented on these phenomena. Also, the application of this flow pattern to industrial processes like rocket motors, cooling by transpiration, chromatography, water treatment, ion exchange and the use of gaseous diffusion for separation of uranium have been the subjects of several investigations [3,6,16-18,22,28,29]. As a result, many methods for handling the generated nonlinear models that usually comes up from the physics of the fluid flow have been coming up to enhance the parametric study of the controlling agents. Recently, Majdalani [16] as well as the tremendous research study by Majdalani and Roh [17] analyzed the oscillatory fluid flow with injection by employing asymptotic formulations. The method has also been used for the purpose of obtaining symbolic oscillatory solutions with arbitrary suction condition [13]. They also analyzed laminar fluid flow through porous system by considering large suction wall parameters using Green transformation [12], while Zhou and Majdalani [32] performed a comparative study by considering the mean flow and regression wall of a rocket motor using a method of asymptotic and finite difference. The obtained numerical results in their work were used to obtain and study the effect of the average velocity as well as the impact of low Reynolds number on the flow dynamics [19]. Nonetheless, the application of multiple scale solutions to the problem in discuss have been explicitly considered in the work of Robinson [23], Zarturska et al. [31] and Si et al. [24,25]. Also, Majdalani et al. [20] made an attempt to obtain the approximate analytical solutions to weak permeable fluid flow. Dinarvand and Rashidi [8] employed homotopy perturbation method in analyzing the multi-dimensional fluid flow with appreciable viscosity along porous rectangular boundaries. Homotopy perturbation method and Homotopy analysis method, usually abbreviated as HPM and HAM respectively, were adopted by Dinarvand $e t$ al. [7] to provide the approximate analytical solutions to Berman's two dimensional fluid flow governing equation in porous passage with wall injection or suction. The authors reported that the developed close form solution via HPM is invalid when considering extreme flow Reynolds numbers. This weakness in the solution of HPM for large Reynolds number is also noticed in the use of the remaining perturbation techniques. Thus, Xu et al. [30] employed HAM to remove this draw back. Although, it is a generally accepted fact that the HAM is efficient and reliable, it suffers from assumptions which generally limit its applications. These assumptions include the fact that the solution should conform to some socalled solution expression law and the principle of coefficient ergodicity. Also, it requires the search for auxiliary and

*Corresponding author (mikegbeminiyi@gmail.com) 
unknown parameters that accelerate the convergence and satisfies the end boundary conditions. Such quests are achieved by indirectly applying numerical methods, thus makes HAM a semi-close form solution instead of total analytical solution. Also in practice, such results with big algebraic terms are not convenient for use by engineers, software developers, scientist and designers [26].

Different reviews have been performed on the application of semi or approximate analytical and numerical schemes. Focusing on the available numerical schemes, the numerical solutions using Pseudospectral collocation method represent efficient ways of obtaining solutions to nonlinear problems even with complexities in the boundary conditions $[1,2,4,5,9-11$, 14,15,21,27]. Chebyshev collocation technique (CPCM) uses Chebyshev polynomials and Chebyshev-Gauss-Lobatto (CGL) or spatial discretization for its approximations. This method transforms integral and differential equations into algebraic form that can easily be solved numerically. CPCM is general, easy to implement and yields very accurate results. It is recognized that this method generates better approximations than most of the existing numerical schemes. Therefore, in this present work, CPCM is utilized to analyze transient two-dimensional viscous fluid flow through porous walls of admittance that are susceptible to expanding/contracting under suction or injection. The results of the CPCM are verified numerically with Runge-Kutta method coupled with the shooing method. Thereafter, the developed solutions are used for the parametric studies, with the results graphically presented, analyzed and discussed.

\section{Formulation of the fluid flow problem}

Consider the unsteady, laminar, fully developed, isothermal, and incompressible fluid flow in a two-dimensional porous channel enclosed by two permeable walls to enable nanofluid passage during successive contractions/expansions. The two-dimensional equations of the continuity and motion are

$$
\begin{gathered}
\frac{\partial \tilde{u}}{\partial \tilde{x}}+\frac{\partial \tilde{v}}{\partial \tilde{y}}=0 \\
\rho_{n f}\left(\frac{\partial \tilde{u}}{\partial \tilde{t}}+\tilde{u} \frac{\partial \tilde{u}}{\partial \tilde{x}}+\tilde{v} \frac{\partial \tilde{u}}{\partial \tilde{y}}\right)=-\frac{\partial \tilde{p}}{\partial \tilde{x}}+\mu_{n f}\left(\frac{\partial^{2} \tilde{u}}{\partial \tilde{x}^{2}}+\frac{\partial^{2} \tilde{u}}{\partial \tilde{y}^{2}}\right)-\sigma B_{o}^{2} \tilde{u} \\
\rho_{n f}\left(\frac{\partial \tilde{v}}{\partial \tilde{t}}+\tilde{u} \frac{\partial \tilde{v}}{\partial \tilde{x}}+\tilde{v} \frac{\partial \tilde{v}}{\partial \tilde{y}}\right)=-\frac{\partial \tilde{p}}{\partial \tilde{y}}+\mu_{n f}\left(\frac{\partial^{2} \tilde{v}}{\partial \tilde{x}^{2}}+\frac{\partial^{2} \tilde{v}}{\partial \tilde{y}^{2}}\right),
\end{gathered}
$$

where

$$
\begin{gathered}
\rho_{n f}=\rho_{f}(1-\phi)+\rho_{s} \phi \\
\mu_{n f}=\frac{\mu_{f}}{(1-\phi)^{2.5}} .
\end{gathered}
$$

The non-slip conditions at the boundaries are

$$
\begin{gathered}
\tilde{y}=a(t), \quad \tilde{u}=0, \quad \tilde{v}=-V_{w}=-\frac{a}{c} ; \\
\tilde{y}=0, \quad \frac{\partial \tilde{u}}{\partial \bar{y}}=0, \quad \tilde{v}=0 ; \\
\tilde{x}=0, \quad \tilde{u}=0,
\end{gathered}
$$

where $c=\frac{\dot{a}}{V_{w}}$ is the suction/injection parameter. Introducing the stream and vorticity functions

$$
\tilde{u}=\frac{\partial \tilde{\psi}}{\partial \bar{y}}, \quad \tilde{v}=\frac{\partial \tilde{\psi}}{\partial \bar{x}} .
$$

Eliminating the pressure term from Equations (2) and (3), the transport equation becomes;

$$
\rho_{n f}\left(\frac{\partial \tilde{\xi}}{\partial \tilde{t}}+\tilde{u} \frac{\partial \tilde{\xi}}{\partial \tilde{x}}+\tilde{v} \frac{\partial \tilde{\xi}}{\partial \tilde{y}}\right)=\mu_{n f}\left(\frac{\partial^{2} \tilde{\xi}}{\partial \tilde{x}^{2}}+\frac{\partial^{2} \tilde{\xi}}{\partial \tilde{y}^{2}}\right)+\sigma B_{o}^{2} \tilde{u} \frac{\partial \tilde{u}}{\partial \tilde{y}}
$$

where

$$
\tilde{\xi}=\frac{\partial \tilde{v}}{\partial \tilde{x}}-\frac{\partial \tilde{u}}{\partial \tilde{y}}
$$

Using the following similarity functions, Equation (8) is converted into an ordinary differential equation;

$$
\tilde{u}=\frac{\mu_{f} x}{\rho_{f} H^{2}} \tilde{f}^{\prime}(\eta, \tilde{t}), \quad \tilde{v}=\frac{-\mu_{f}}{\rho_{f} H} \tilde{f}(\eta, \tilde{t})
$$


where

$$
\eta=\frac{\tilde{y}}{H}, \quad \tilde{f}^{\prime}(\eta, \tilde{t})=\frac{\partial \tilde{f}(\eta, \tilde{t})}{\partial \eta} .
$$

If one substitutes Equation (9) and (10) into Equation (8), Equation (11) is obtained as expressed below

$$
\tilde{f}_{\eta \eta \eta \eta}+\alpha(t)\left(\eta \tilde{f}_{\eta \eta \eta}+3 f_{\eta \eta}\right)+\tilde{f} \tilde{f}_{\eta \eta \eta}-\tilde{f}_{\eta} \tilde{f}_{\eta \eta}-\frac{\rho_{n f} a^{2}}{\mu_{n f}} \tilde{f}_{\eta \eta t}-H a^{2} \tilde{f}_{\eta \eta}=0
$$

subject to the boundary conditions expressed as

$$
\begin{gathered}
\eta=0, \quad \tilde{f}=0, \quad \tilde{f}_{\eta \eta}=0 ; \\
\eta=1, \quad \tilde{f}=R e, \quad \tilde{f}_{\eta}=0
\end{gathered}
$$

where

$$
\alpha(\tilde{t})=\frac{\rho_{f} a \dot{a}(\tilde{t})}{\mu_{f}}
$$

is the rate wall dilation in dimensionless form which is negative for contraction and positive for expansion, while

$$
R e=\frac{\rho_{f} a V_{w}}{\mu_{f}}
$$

is the Reynolds number of permeation. Note that

$$
\psi=\frac{\tilde{\psi}}{a \dot{a}}, \quad u=\frac{\tilde{u}}{\dot{a}}, \quad v=\frac{\tilde{v}}{\dot{a}}, \quad f=\frac{\tilde{f}}{R e} \Rightarrow \psi=\frac{x f}{c}, \quad u=\frac{x f^{\prime}}{c}, \quad v=\frac{-f}{c}, \quad c=\frac{\alpha}{R e}
$$

Equations (11) and (12) normalized to

$$
f_{\eta \eta \eta \eta}+(1-\phi)^{2.5}\left((1-\phi)+\frac{\rho_{s}}{\rho_{f}} \phi\right)\left\{\alpha(t)\left(\eta f_{\eta \eta \eta}+3 f_{\eta \eta}\right)+R e\left(f f_{\eta \eta \eta}-f_{\eta} f_{\eta \eta}\right)-\frac{\rho_{f} a^{2}}{\mu_{f}} f_{\eta \eta t}\right\}-H a^{2} f_{\eta \eta}=0,
$$

subject to

$$
\begin{array}{ll}
\eta=0, & f=0, \quad f_{\eta \eta}=0 ; \\
\eta=1, & f=1, \quad f_{\eta}=0 .
\end{array}
$$

Assuming $\alpha(\tilde{t})=\frac{\rho_{f} a \dot{a}(\tilde{t})}{\mu_{f}}$ is constant and $f=f(\eta)$, then $f_{\eta \eta t}=0$ and Equation (14) reduces to

$$
f_{\eta \eta \eta \eta}+(1-\phi)^{2.5}\left((1-\phi)+\frac{\rho_{s}}{\rho_{f}} \phi\right)\left\{\alpha(\tilde{t})\left(\eta f_{\eta \eta \eta}+3 f_{\eta \eta}\right)+R e\left(f f_{\eta \eta \eta}-f_{\eta} f_{\eta \eta}\right)\right\}-H a^{2} f_{\eta \eta}=0
$$

and the conditions remain unchanged. If $\alpha=0$ is substituted into Equation (16), the classical Berman's model [3] for stationary walls channels is recovered.

\section{Solution method: Chebyshev pseudo-spectral collocation method (CPCM)}

Equation (16) is a non-linear ordinary differential equation which is to be solved alongside with boundary conditions in Equation (15). In order to solve the nonlinear equation, CPCM is applied. Following the method procedures, the $\eta$ direction domain is approximated by $[0, L]$, where $\mathrm{L}$ is the edge of the boundary layer. Using the algebraic mapping $\eta=(\gamma+1) L / 2$, the physical region can be mapped out into Chebyshev collocation method domain, usually [-1, 1]. The unknown variable in Equation (16) is approximated using the following truncated series of polynomials:

$$
f(\gamma)=\sum_{k=0}^{N} \widehat{f}_{k} T_{k}(\gamma)
$$

where the values of $\widehat{f}_{k}$ are the coefficient of the series to be determined, $T_{k}$ is the Chebychev polynomial of degree $k$ which is defined on the interval $\gamma \in[-1,1]$.

$$
T_{k}(\gamma)=\cos \left(k \cos ^{-1} \gamma\right) ; \quad k=0,1,2, \cdots
$$

The application of pseudospectral collocation method requires that the discretization of the space dimensionless models be carried out through the utilization of Chebyshev-Lobatto collocation points. Using the Gauss-Lobatto collocation points, we define the Chebyshev nodes in the interval $[-1,1]$ as

$$
\gamma_{j}=\cos \frac{\pi j}{N} ; \quad-1 \leq \gamma \leq 1, j=0,1, \cdots, N
$$


The derivative of the functions $f$ is represented as

$$
\frac{d f}{d \eta}=\frac{2}{L} \sum_{k=0}^{N} \mathbf{D}_{j k} \widehat{f}_{k}
$$

Where $\mathbf{D}$ is the Chebyshev spectral method differentiation matrix. Higher order derivatives are computed as multiple powers of $\mathbf{D}$, i.e.

$$
\frac{d^{i} f}{d \eta^{i}}=\left(\frac{2}{L}\right)^{i} \sum_{k=0}^{N} \mathbf{D}_{j k}^{i} \widehat{f}_{k} ; \quad k=0,1,2, \cdots, N,
$$

where $i$ is the order of the derivative. By using Equations (17) - (21) in the models and the conditions in Equation (16), the following equations are obtained

$$
\overline{\mathbf{D}}^{4} \mathbf{F}+(1-\phi)^{2.5}\left((1-\phi)+\frac{\rho_{s}}{\rho_{f}} \phi\right)\left\{\alpha(\tilde{t})\left(\eta \overline{\mathbf{D}}^{3} \mathbf{F}+3 \overline{\mathbf{D}}^{2} \mathbf{F}\right)+\operatorname{Re}\left(\mathbf{F} \overline{\mathbf{D}}^{3} \mathbf{F}-(\overline{\mathbf{D}} \mathbf{F})\left(\overline{\mathbf{D}}^{2} \mathbf{F}\right)\right)\right\}-H a^{2} \overline{\mathbf{D}}^{2} \mathbf{F}=0,
$$

where $\overline{\mathbf{D}}=\left(\frac{2}{L}\right) \mathbf{D}, \mathbf{F}=\left\{\widehat{f}_{0}, \widehat{f}_{1}, \ldots, \widehat{f}_{N}\right\}^{T}$ and the superscript $T$ denotes the transpose. Also, the boundary conditions become

$$
\begin{array}{r}
\widehat{f}_{N}=0, \quad \sum_{k=0}^{N} \widehat{\mathbf{D}}_{N k}^{2} \widehat{f}_{k}=0, \\
\sum_{k=0}^{N} \widehat{f}_{N k}=1, \quad \sum_{k=0}^{N} \widehat{\mathbf{D}}_{N k} \widehat{f}_{k}=0 .
\end{array}
$$

Although, the algorithm for the implementation of the CPCM is given in the next section, it should be stated that Equation (22) can be solved separately subject to the boundary conditions in Equation (23) using a MATLAB nonlinear equation solver "fzero" which is based on the quasi-Newton method.

\subsection{Algorithm for the implementation of the CPCM}

The CPCM can be implemented as follows:

Step 1: Input the number of collocation points, and compute the coordinate values of the nodes for the matrices of the first-order, second-order, third-order and fourth-order derivatives.

Step 2: Set the dimensionless velocity at the initial values in all directions except at the boundaries.

Step 3: Use Equation (22), assemble the matrix.

Step 4: Impose the boundary conditions in Equation (23) in the algebraic systems of equations and solve the equation directly.

Step 5: Using the convergence criteria $\sum\left|\phi_{i}^{p}-\phi^{p-1}\right| \leq 10^{-12}$, terminate the iteration procedure, otherwise, return to Step 3 .

\section{Results and discussion}

The verification of the approximate analytical solution through CPCM is carried out as shown in Table 1 . The comparisons of the results through the two different schemes are depicted in Table 1 and Table 2 . The analysis revealed that the results obtained by CPCM are in excellent agreements with the results obtained by the numerical method. This shows that the results of CPCM are completely reliable and physically realistic as the efficiency and accuracy of CPCM is demonstrated.

Also, the established solutions are employed in the parametric study of the flow controlling variables during contraction or expansion through porous channel with suction and injection. The Reynold number (Re) is represented as " $R$ " in the figures. 


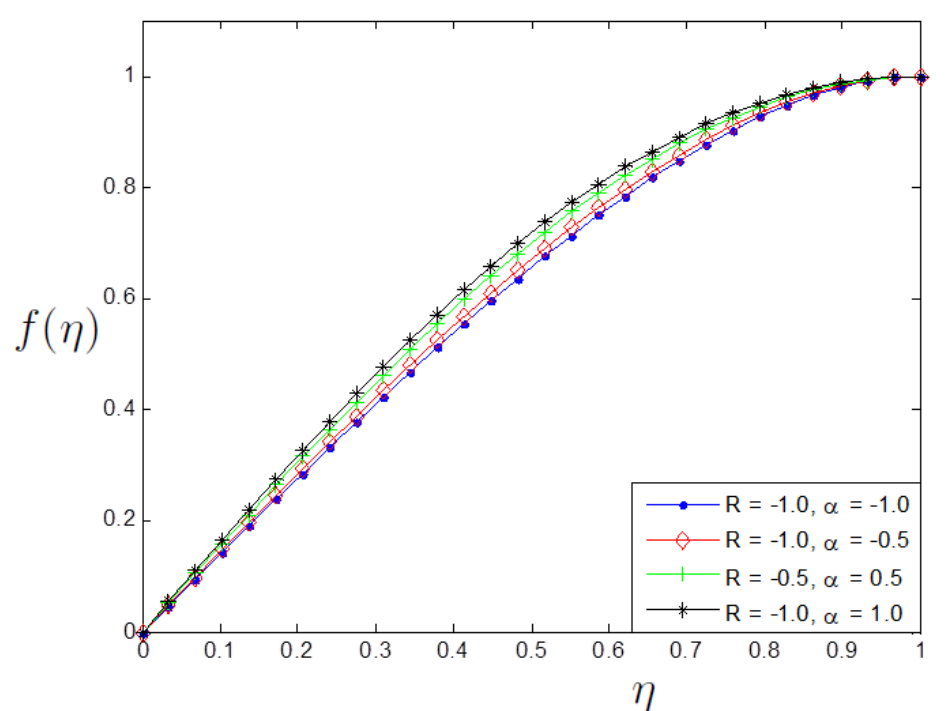

(a)

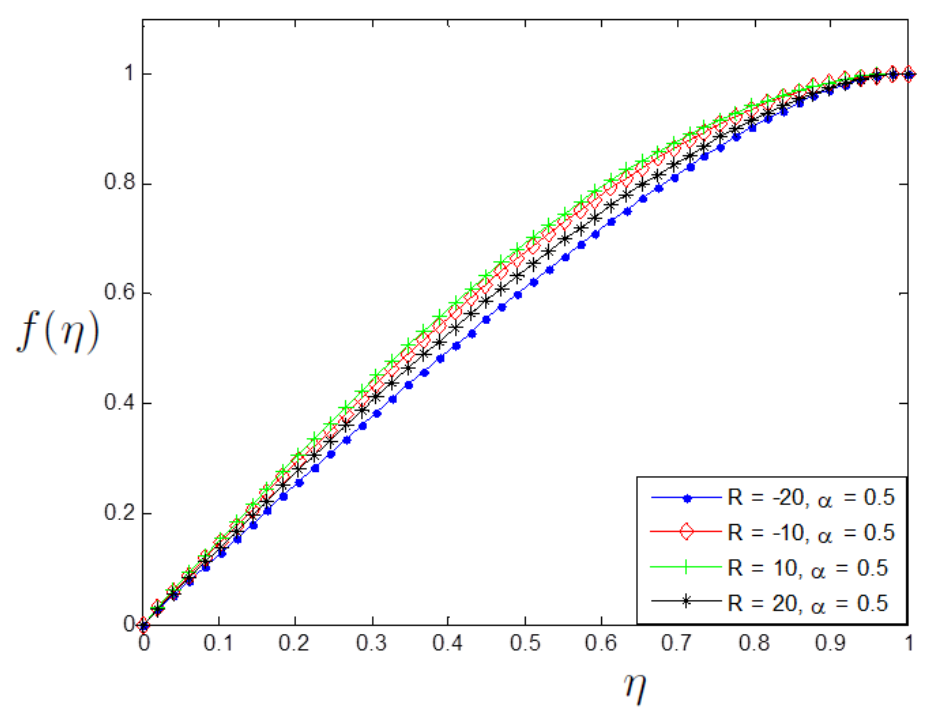

(c)

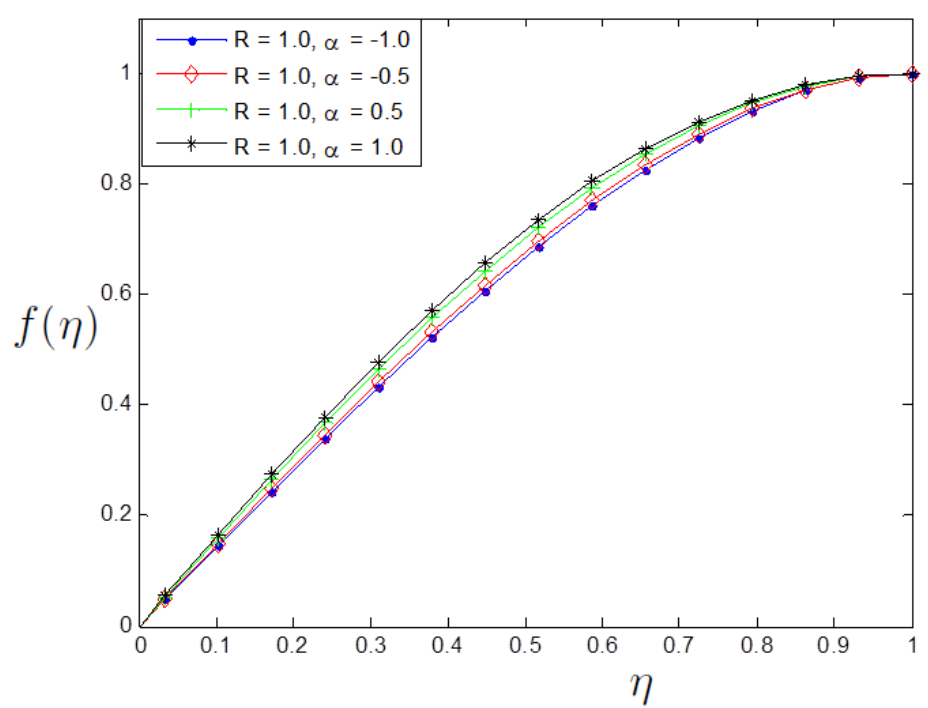

(b)

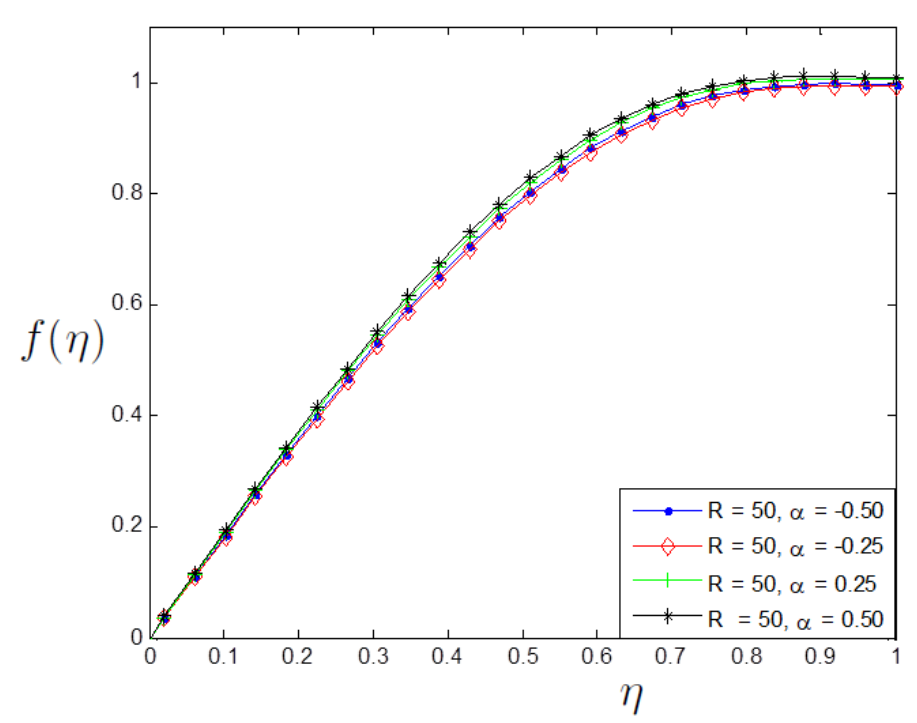

(d)

Figure 1: Variation of $f(\eta)$ for different expansion and contraction ratio, $\alpha$ and different small values of Reynold number.

Table 1: Comparison of the results concerning the flow for small Reynolds number for suction and expansion.

\begin{tabular}{ccc}
\hline \multicolumn{3}{c}{$f(\eta)$} \\
Re $=5.0, \alpha=0.5$ \\
\hline$\eta$ & $\mathrm{NM}$ & CPCM \\
\hline 0.0 & 0.000000 & 0.000000 \\
0.1 & 0.152874 & 0.152879 \\
0.2 & 0.301551 & 0.301554 \\
0.3 & 0.442606 & 0.442608 \\
0.4 & 0.573196 & 0.573191 \\
0.5 & 0.690876 & 0.690873 \\
0.6 & 0.793373 & 0.793378 \\
0.7 & 0.878373 & 0.878375 \\
0.8 & 0.943297 & 0.943293 \\
0.9 & 0.985090 & 0.985094 \\
1.0 & 1.000000 & 1.000000
\end{tabular}




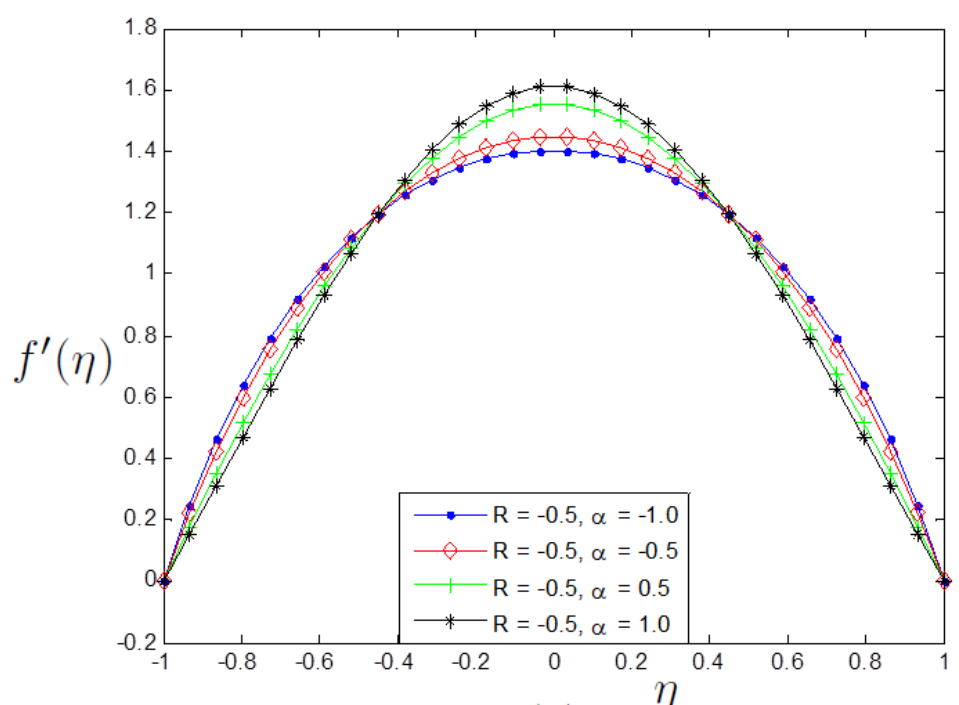

(a)

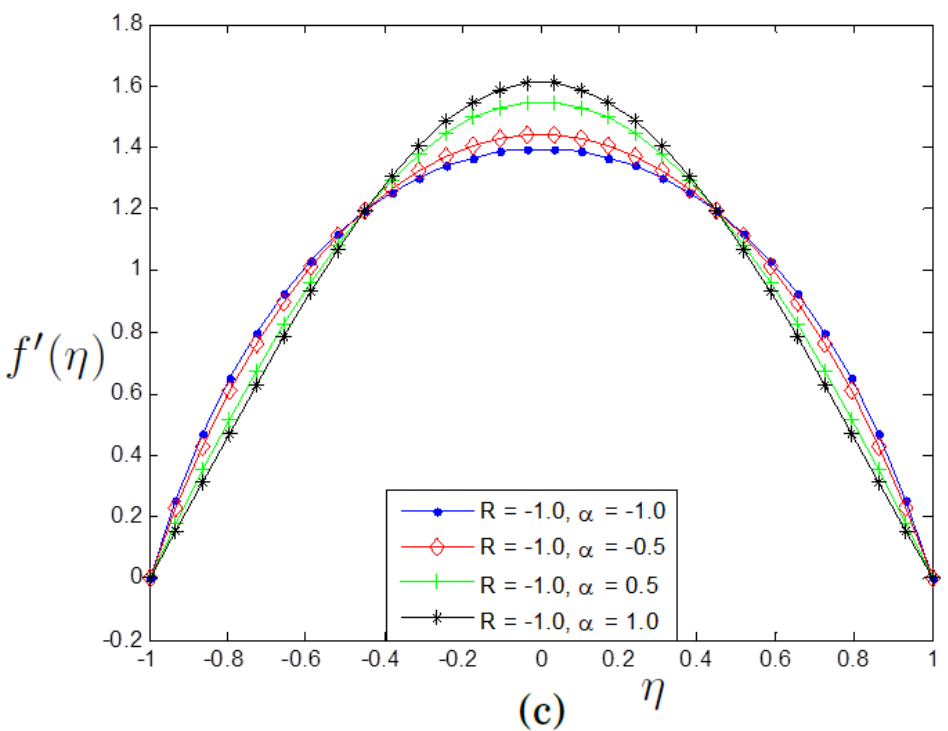

(c)

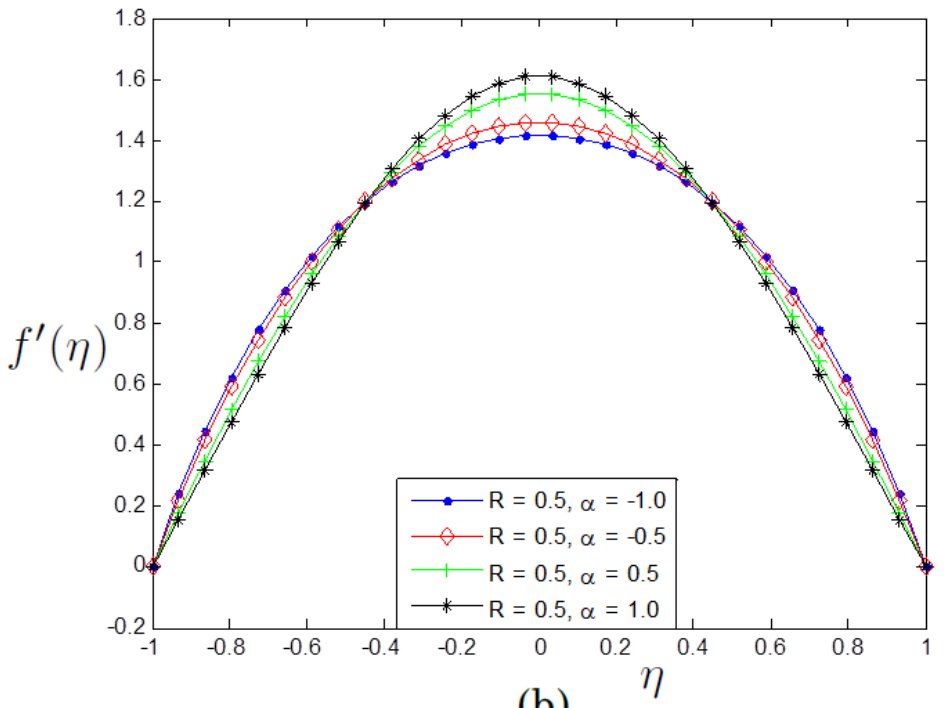

(b)

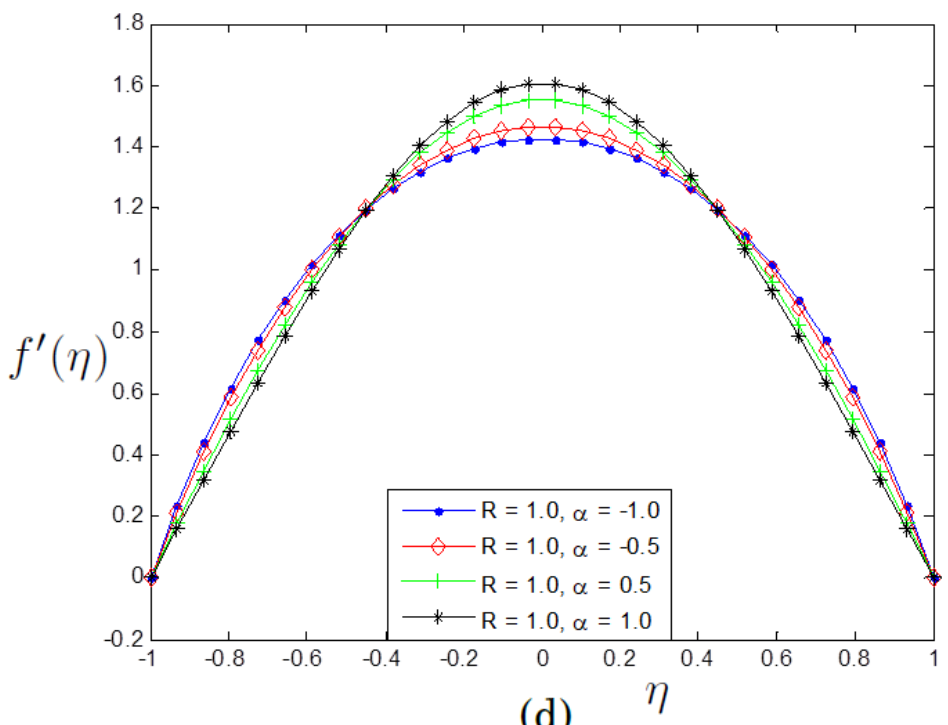

(d)

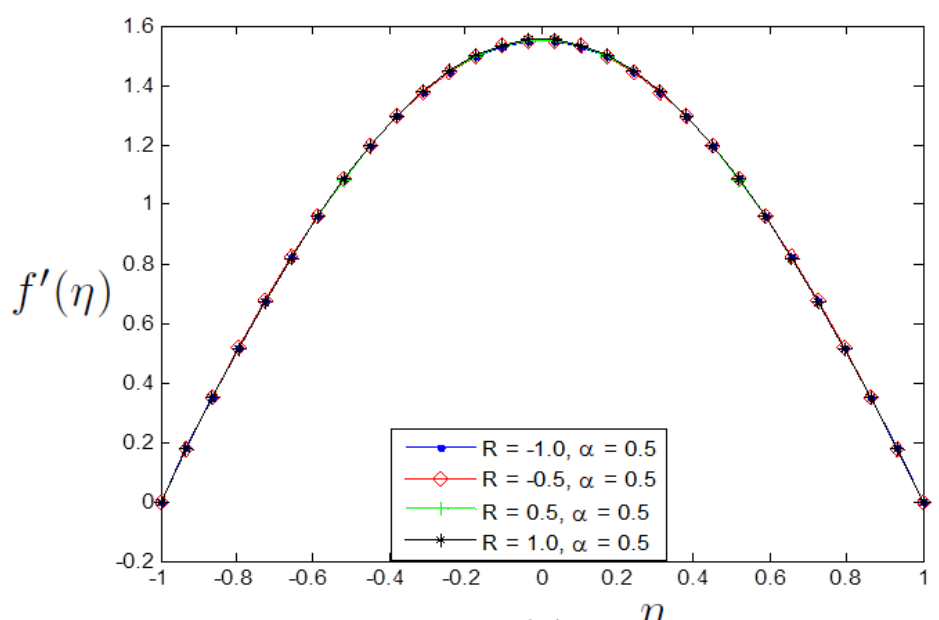

(e)

Figure 2: Variation of $f^{\prime}(\eta)$ for different expansion and contraction ratio, $\alpha$ and Reynold number. 
Table 2: Comparison of the results regarding the flow for small Reynolds number for injection and expansion.

\begin{tabular}{ccc}
\hline \multicolumn{3}{c}{$f^{\prime}(\eta)$} \\
Re $=5.0, \alpha=0.5$ \\
\hline$\eta$ & NM & CPCM \\
\hline 0.0 & 1.536154 & 1.536157 \\
0.1 & 1.151411 & 1.151415 \\
0.2 & 1.453855 & 1.453859 \\
0.3 & 1.362554 & 1.362558 \\
0.4 & 1.245207 & 1.245203 \\
0.5 & 1.104631 & 1.104637 \\
0.6 & 0.941489 & 0.941483 \\
0.7 & 0.754210 & 0.754214 \\
0.8 & 0.539188 & 0.539182 \\
0.9 & 0.290458 & 0.290454 \\
1.0 & 0.000000 & 0.000000
\end{tabular}

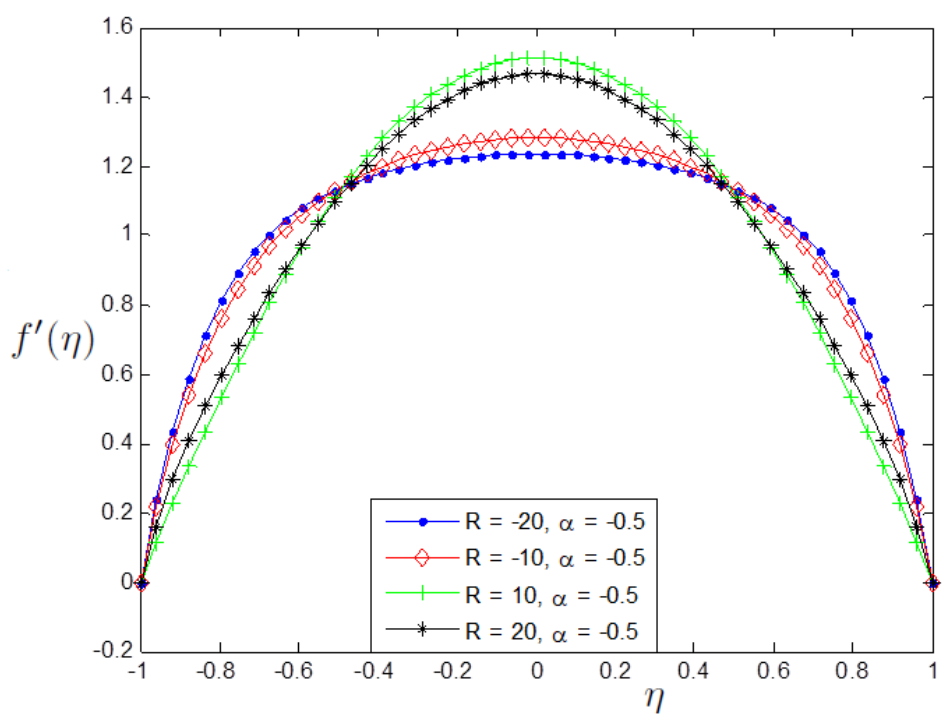

(a)

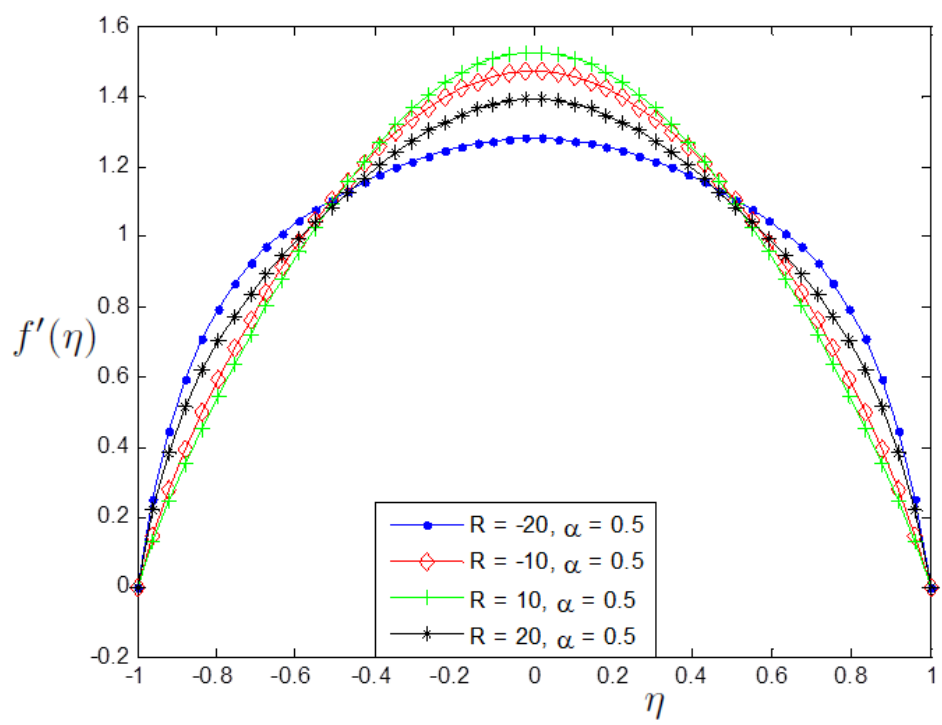

(b)

Figure 3: Variation of $f^{\prime}(\eta)$ for different expansion and contraction ratio, $\alpha$ and Reynold number.

Effects of the Reynolds number of permeation and dimensionless rate of dilation on mean fluid velocities are depicted in Figure 1. Effects of $R e$, on dimensionless axial velocity are displayed in Figures 1(a)-(d), 2(a)-(d), 3(a) and 3(b). The figures illustrate that the expansion process and increase in Re reduce the channel center axial velocity. Also, this velocity reduces at the channel middle but increases towards the permeable walls due to an increase in the wall expansion ratio. Figures 2(a)-(d) show the behavior of axial velocity for different permeation Reynolds number, over a range of dimensionless rate of wall dilation. The figures show that, for every suction or injection, the velocity is optimal at the middle of the porous channel. The reason is because the fluid flow around this domain becomes difficult to make up due to the space already created by the wall expansion. As the expansion ratio of the permeable wall increases, the domain centre velocity reduces except near the wall where an increase is noticed. Similarly, as presented in Figures 2(a)-(d) and 3(a), a reduced axial velocity can be achieved when desired by simply increasing the system contraction ratio. However, it turns out to be greater around the wall due to the increased mobility of the viscous flow. Hence, both suction and expansion can be used to augment fluid flow velocity through the channel. The results obtained in the present work when considering expanding wall show that for every injection or suction, expansion ratio is directly proportional to mean lateral velocity only near the center and inversely proportional around the walls.

\section{Conclusion}

In this work, numerical investigations of unsteady two-dimensional viscous fluid flow in porous medium through contracting/expanding boundaries with suction/injection have been carried out using CPCM. From the parametric studies, an increase in the flow $R e$ reduces the centre axial velocity for the case of expansion while an antonymous effect is demonstrated for the case of contraction. Also, as the wall expansion ratio increases, the velocity at the center decreases and increases near the wall. When considering expanding walls, increasing expansion ratio leads to increased axial velocity 
near the center and the decreased axial velocity near the walls. It is hoped that the work will help and advance the understanding the laminar flow of nanofluid in a porous channel through slowly expanding/contracting walls with injection or suction.

\section{Nomenclature}

$\dot{a}$ time-dependent rate

$B_{0}$ electromagnetic induction

Ha Hartmann number

$\bar{p}$ Pressure

Re permeation Reynolds number

$t$ time

$\bar{u}$ velocity component in $x$-direction

$\bar{v}$ velocity component in $y$-direction

$V_{w}$ fluid inflow velocity at the wall

$\bar{x}$ coordinate axis parallel to the channel walls

$\bar{y}$ coordinate axis perpendicular to the channel walls

$\rho_{n f}$ density of the nanofluid

$\rho_{f}$ density of the fluid

$\mu_{n f}$ dynamic viscosity of the nanofluid

$\rho_{s}$ density of the nanoparticles

$\phi$ fraction of nanoparticles in the nanofluid

$\sigma$ electrical conductivity

$\alpha$ dimensionless wall dilation rate

\section{Acknowledgment}

The authors acknowledge the moral and materials supports from University of Lagos, Nigeria.

\section{References}

[1] A. Alexandrescu, A. B. Orovio, J. R. Salgueiro, V. M. Garcia, Mapped Chebyshev pseudospectral method for the study of multiple scale phenomena, Comput. Phys. Commun. 180 (2009) 912-919.

[2] A. Bayliss, E. Turkel, Mappings and accuracy for Chebyshev psedo-spectral approximations, J. Comput. Phys. 101 (1992) $349-359$.

[3] A. S. Berman, Laminar flow in channels with porous walls, J. Appl. Phys. 24 (1953) 1232-1235.

[4] E. A. Butcher, O. A. Bobrenkov, On the Chebyshev spectral continuous time approximation for constant and periodic delay differential equations, Commun. Nonlinear Sci. Numer. Simul. 16 (2011) 1541-1554.

[5] C. Canuto, M. Y. Hussaini, A. Quarteroni, T. Zang, Spectral Method in Fluid Dynamics, Springer, New York, 1998.

[6] E. C. Dauenhauer, J. Majdalani, Exact self-similarity solution of the Navier-Stokes equations for a porous channel with orthogonally moving walls, Phys. Fluids 15 (2003) 1485-1495.

[7] S. Dinarvand, A. Doosthoseini, E. Doosthoseini, M. M. Rashidi, Comparison of HAM and HPM methods for Berman's model of two-dimensional viscous flow in porous channel with wall suction or injection, Adv. Theor. Appl Mech. 1 (2008) 337-347.

[8] S. Dinarvand, M. M. Rashidi, A reliable treatment of a homotopy analysis method for two dimensional viscous flow in a rectangular domain bounded by two moving porous walls, Nonlinear Anal. Real World Appl. 11 (2010) 1502-1512.

[9] A. D. Dinu, R. M. Botez, I. Cotoi, Chebyshev polynomials for unsteady aerodynamic calculations in aeroservoelasticity, J. Aircr. 43 (2006) $165-171$.

[10] F. Fahroo, I. M. Ross, Direct trajectory optimization by a chebyshev pseudospectral method, J. Guid. Control Dynam. 25 (2002) 160-166.

[11] A. K. Gopinath, A. Jameson, Time Spectral Method for Periodic Unsteady Computations over Two- and Three-Dimensional Bodies, 43rd AIAA Aerospace Sciences Meeting and Exhibit, 2005

[12] T. A. Jankowski, J. Majdalani, Laminar flow in a porous channel with large wall suction and a weakly oscillatory pressure, Phys. Fluids 14 (2002) $1101-1110$.

[13] T. A. Jankowski, J. Majdalani, Symmetric solutions for the oscillatory channel flow with arbitrary suction, J. Sound Vib. 294 (2006) 880-893.

[14] A. H. Khater, R. S. Temsah, M. M. Hassan, A Chebyshev spectral collocation method for solving burgers-type equations, J. Comput. Appl. Math. 222 (2008) 333-350.

[15] D. Kosloff, H. Tal-Ezer, A modified Chebyshev pseudospectral method with an $O\left(N^{-1}\right)$ time step restriction, J. Comput. Phys. 104 (1993) $457-469$.

[16] J. Majdalani, The oscillatory channel flow with arbitrary wall injection, Z. Angew. Math. Phys. 52 (2001) 33-61.

[17] J. Majdalani, T.-S. Roh, The oscillatory channel flow with large wall injection, Proc. Roy. Soc. Lond. Series A 456 (2000) $1625-1657$.

[18] J. Majdalani, W. K. van Moorhem, Multiple-scales solution to the acoustic boundary layer in solid rocket motors, J. Propul. Power 13 (1997) $186-193$.

[19] J. Majdalani, C. Zhou, Moderate-to-large injection and suction driven channel flows with expanding or contracting walls, Z. Angew. Math. Mech. 83 (2003) 181-196.

[20] J. Majdalani, C. Zhou, C. A. Dawson, Two-dimensional viscous flow between slowly expanding or contracting walls with weak permeability, J. Biomech. 35 (2002) 1399-1403.

[21] J. Niu, L. Zheng, Y. Yang, C. Shu, Chebyshev spectral method for unsteady axisymmetric mixed convection heat transfer of power law fluid over a cylinder with variable transport properties, Int. J. Numer. Anal. Model. 11 (2014) 525-540.

[22] L. Oxarango, P. Schmitz, M. Quintard, Laminar flow in channels with wall suction or injection: a new model to study multi-channel filtration systems, Chem. Eng. Sci. 59 (2004) 1039-1051.

[23] W. A. Robinson, The existence of multiple solutions for the laminar flow in a uniformly porous channel with suction at both walls, J. Eng. Math. 10 (1976) 23-40. 
[24] X. H. Si, L. C. Zheng, X. X. Zhang, Y. Chao, Existence of multiple solutions for the laminar flow in a porous channel with suction at both slowly expanding or contracting walls, Int. J. Miner. Metal. Mater. 11 (2011) 494-501.

[25] X. H. Si, L. C. Zheng, X. X. Zhang, M. Li, J. H. Yang, Y. Chao, Multiple solutions for the laminar flow in a porous pipe with suction at slowly expanding or contracting wall, Appl. Math. Comput. 218 (2011) 3515-3521.

[26] M. G. Sobamowo, Thermal analysis of longitudinal fin with temperature-dependent properties and internal heat generation using Galerkin's method of weighted residual, Appl. Therm. Eng. 99 (2016) 1316-1330.

[27] T. W. Tee, L. N. Trefethen, A rational spectral collocation method with adaptively transformed Chebyshev grid points, SIAM J. Sci. Comput. 28 (2006) 1798-1811.

[28] R. M. Terrill, Laminar flow in a uniformly porous channel, Aeronaut. Quart. 15 (1964) 299-310.

[29] R. M. Terrill, Laminar flow in a uniformly porous channel with large injection, Aeronaut. Quart. 16 (1965) $323-332$.

[30] J. Xu, Z. L. Lin, S. J. Liao, J. Z. Wu, J. Majdalani, Homotopy based solutions of the Navier-Stokes equations for a porous channel with orthogonally moving walls, Phys. Fluids 22 (2010) Art\# 053601.

[31] M. B. Zaturska, P. G. Drazin, W. H. H. Banks, On the flow of a viscous fluid driven along a channel by suction at porous walls, Fluid Dyn. Res. 4 (1988) 151-178.

[32] C. Zhou, J. Majdalani, Improved mean-flow solution for slab rocket motors with regressing walls, J. Propul. Power 18 (2002) $703-711$. 